九州大学学術情報リポジトリ

Kyushu University Institutional Repository

\title{
Scouting Strategy for Biasing Fireworks Algorithm Search to Promising Directions
}

Yu, Jun

Graduate School of Design, Kyushu University

Tan, Ying

School of Electronics Engineering and Computer Science, Peking University

Takagi, Hideyuki

Faculty of Design, Kyushu University

ht tp://hdl. handle. net/2324/1916247

出版情報 : 2018-07-31. ACM

バージョン：

権利関係 : 


\section{Scouting Strategy for Biasing Fireworks Algorithm Search to Promising Directions}

\author{
Jun YU \\ Graduate School of Design \\ Kyushu University, Fukuoka, Japan \\ yujun(AT)kyudai.jp
}

\author{
Ying TAN \\ School of Electronics Engineering and \\ Computer Science \\ Peking University, Peking, China \\ ytan(AT)pku.edu.cn
}

\author{
Hideyuki TAKAGI \\ Faculty of Design \\ Kyushu University, Fukuoka, Japan \\ takagi(AT)design.kyushu-u.ac.jp
}

\begin{abstract}
We propose a scouting strategy to find better searching directions in fireworks algorithm (FWA) to enhance its exploitation capability. It generates spark individuals from a firework individual one by one by checking if the generated spark climbs up to a better direction, and this process continues until spark individual climbing down is generated, while canonical FWA generates spark individuals around a firework individual at once. We can know potential search directions from the number of consciously climbing up sparks. Besides this strategy, we use a filtering strategy for a random selection of FWA, where worse sparks are eliminated when their fitness is worse than their parents, i.e. fireworks, and become unable to survive in the next generation. We combined these strategies with the enhanced FWA (EFWA) and evaluated using 28 CEC2013 benchmark functions. Experimental results confirm that the proposed strategies are effective and show better performance in terms of convergence speed and accuracy. Finally, we analyze their applicability and provide some open topics.
\end{abstract}

Keywords-swarm intelligence, fireworks algorithm, filtering selection, multi-directional trackings, scouting strategy

\section{INTRODUCTION}

Evolutionary computing (EC) has attracted academies and industries due to its various excellent characteristics, such as high robustness and wide applicability. Many novel and effective ideas have been introduced to various EC algorithms, new EC variations with excellent performance have been developed, e.g. fireworks algorithm (FWA) [1] used in this paper. Although these latecomers have achieved remarkable performance, few algorithms focus on understanding the fitness landscape and ignore its characteristics.

The main objective of this paper is to propose a new strategy to further enhance exploitation capability of individuals by tracking one or more directions on a fitness landscape according to their potential. To evaluate its performance, we combine it with the enhanced FWA (EFWA) [2] and applied it to 28 CEC2013 benchmark functions. Furthermore, a filtering strategy is merged into random selection not to waste search resources.

\section{FIREWORKS ALGORITHM}

Firework is launched into the sky, and many sparks are generated around it. We can compare the firework explosion to computational search; sparks are compared to local searching points around a specific point (firework), and fireworks are compared to global search. FWA simulates the explosion process iteratively to find the optimal solution, which mainly consists of three operations: explosion, mutation and selection.

\section{Two Proposed Strategies}

Scouting Strategy: Canonical FWA (1) determines the number of spark individuals and their searching area size around each firework individual, (2) generates sparks randomly in the area at once, and (3) generates a mutant spark individual(s), and (4) determines new fireworks in the next generation using information of each firework and its sparks. Since sparks are generated at once in the step (2), their information cannot be fully used.

To improve this point, our scouting strategy generates spark individuals one by one by checking their fitness. This trace looks like a scout soldier finding a better direction. When the $n$-th spark individual become worse than the $(n-1)$-th spark, the continuous scouting stops and new scouting starts from the initial point, the coordinates of their firework. Since the number of sparks continuous generated or the scouting depth is an index of better local search direction, we can use it that cannot be obtain in the step (2) of the canonical FWA. Due to this mechanism, a search area size of spark individuals is not limited to the fixed size in the step (1) but can extend to a better direction using the same number of sparks.

Filtering Strategy: The second improvement is to strengthen the competition among individuals. All spark individuals of conventional FWA have an opportunity to be selected as individuals in the next generation regardless their superiority to their parents, i.e. firework individuals. It may lead to repeatedly explore in poor areas and result in waste of searching resources.

Filtering strategy uses the fitness of a firework individual as a criterion for filtering its poor sparks and keeping its good sparks. After then, reserved sparks, mutation sparks and current fireworks are mixed together and used to be selected for the next generation. This strategy can increase the probability of searching in potential areas and accelerate FWA evolution to promising directions.

\section{EXPERIMENTAL EVALUATIONS}

We evaluate proposed strategies using 28 functions from the CEC2013 benchmark test suite [3] $\times 3$ dimensional 
TABLE I: Wilcoxon signed-rank test results for EFWA vs. Proposal (EFWA + proposed strategies) at the stop condition. $\gg$ and $>$ mean that the left is better than the right with significance levels of $1 \%, 5 \%$ respectively, and $\approx$ means there is no significance.

\begin{tabular}{|c|c|c|c|}
\hline Func. & $2-D$ & $10-D$ & $30-D$ \\
\hline$F_{1}$ & Proposal $\gg$ EFWA & EFWA > Proposal & EFWA $\gg$ Proposal \\
\hline$F_{2}$ & Proposal $\approx$ EFWA & Proposal > EFWA & Proposal $\gg$ EFWA \\
\hline$F_{3}$ & Proposal $\approx$ EFWA & Proposal $\gg$ EFWA & Proposal $\gg$ EFWA \\
\hline$F_{4}$ & Proposal > EFWA & Proposal > EFWA & Proposal > EFWA \\
\hline$F_{5}$ & Proposal $\gg$ EFWA & Proposal $\gg$ EFWA & Proposal $\approx$ EFWA \\
\hline$F_{6}$ & Proposal $\approx$ EFWA & Proposal > EFWA & Proposal $\gg$ EFWA \\
\hline$F_{7}$ & EFWA $\approx$ Proposal & Proposal $\gg$ EFWA & Proposal $\gg$ EFWA \\
\hline$F_{8}$ & EFWA $\approx$ Proposal & Proposal $\approx$ EFWA & Proposal $\approx$ EFWA \\
\hline$F_{9}$ & Proposal > EFWA & Proposal $\gg$ EFWA & Proposal $\approx$ EFWA \\
\hline$F_{10}$ & Proposal $\gg$ EFWA & Proposal $\gg$ EFWA & Proposal $\approx$ EFWA \\
\hline$F_{11}$ & EFWA $\approx$ Proposal & Proposal $\gg$ EFWA & Proposal 》EFWA \\
\hline$F_{12}$ & EFWA $\approx$ Proposal & Proposal $\gg$ EFWA & Proposal $\gg$ EFWA \\
\hline$F_{13}$ & Proposal $\approx$ EFWA & Proposal $\gg$ EFWA & Proposal $\gg$ EFWA \\
\hline$F_{14}$ & EFWA $\approx$ Proposal & Proposal $\gg$ EFWA & Proposal $\gg$ EFWA \\
\hline$F_{15}$ & Proposal $\approx$ EFWA & EFWA $\approx$ Proposal & EFWA $\approx$ Proposal \\
\hline$F_{16}$ & Proposal $\approx$ EFWA & EFWA $\gg$ Proposal & EFWA $\gg$ Proposal \\
\hline$F_{17}$ & Proposal > EFWA & Proposal $\gg$ EFWA & Proposal $\gg$ EFWA \\
\hline$F_{18}$ & Proposal > EFWA & Proposal > EFWA & Proposal > EFWA \\
\hline$F_{19}$ & Proposal $\gg$ EFWA & Proposal $\gg$ EFWA & Proposal $\gg$ EFWA \\
\hline$F_{20}$ & Proposal $\approx$ EFWA & Proposal $\gg$ EFWA & EFWA $\gg$ Proposal \\
\hline$F_{21}$ & EFWA $\approx$ Proposal & EFWA $\approx$ Proposal & Proposal $\approx$ EFWA \\
\hline$F_{22}$ & Proposal $\approx$ EFWA & Proposal $\gg$ EFWA & Proposal $\gg$ EFWA \\
\hline$F_{23}$ & Proposal $\approx$ EFWA & Proposal $\approx$ EFWA & EFWA $\approx$ Proposal \\
\hline$F_{24}$ & Proposal $\approx$ EFWA & Proposal $\gg$ EFWA & Proposal $\gg$ EFWA \\
\hline$F_{25}$ & EFWA $\approx$ Proposal & Proposal $\gg$ EFWA & Proposal $\gg$ EFWA \\
\hline$F_{26}$ & EFWA $\approx$ Proposal & Proposal $\gg$ EFWA & Proposal $\gg$ EFWA \\
\hline$F_{27}$ & EFWA $\approx$ Proposal & Proposal $\gg$ EFWA & Proposal > EFWA \\
\hline$F_{28}$ & Proposal $\approx$ EFWA & Proposal $\gg$ EFWA & Proposal 》EFWA \\
\hline
\end{tabular}

settings of $D=2,10$ and $30 \times 30$ trial runs. We apply the Wilcoxon signed-rank test to the fitness values at the stop conditions, 1,000, 10,000, and 40,000 fitness calculations for $2-D, 10-D$, and 30- $D$, respectively, and check the significance between EFWA and (EFWA + proposed strategies). Table I shows the statistical test results.

\section{Discussions}

We propose a scouting strategy to track the current promising directions to dig deeper potential areas instead of randomly exploring within a fixed range assigned to each firework individual, which means that the better area is, the deeper and more careful exploitation is conducted. The proposals do not change the number of resources allocated to each firework individual, i.e. there is no extra fitness consumptions. Generated sparks also have an opportunity to produce new sparks to increase population diversity. Although we simply used single point tracking to explore the local information as our first attempt, we also can use multi-point tracking, generating multiple points rather than a point, to reduce the risk of single point tracking, i.e. falling into local optima.

The main contribution of the second strategy is to increase the probability of exploring potential areas and avoid ineffective searches. In the conventional EFWA, even though some sparks are worse than their parent (firework), they have a chance to remain in the next generation. If such sparks are selected, they may search in less potential areas, waste resources, which results slow convergence. The second strategy filters poor sparks and ensures to make the whole population evolve toward the optima areas.

Applicability of our proposed strategies is also a feature. They are appicable to not only EFWA used in this paper but also any other variants of FWA. Furthermore, these two proposals can be combined with various EC algorithm individually or in combination.

Finally, to analyze their performances, Wilcoxon signedrank test was applied to fitness values at the stop conditions and checked significance between EFWA and (EFWA + proposed strategies). The test results in Table I shows that the proposed strategies can improve the performance of the conventional EFWA significantly in most cases, and the proposed strategies resulted faster convergence. This effect was obvious for complex task, i.e. higher dimensional tasks. It may be because the total number of sparks is not sufficient for the complex highdimensional problems and using tracking explosions instead of explosion in a fixed range can learn more quickly about local fitness information.

\section{CONCLUSION}

We proposed two strategies to enhance local exploitation capabilities. The first strategy focuses on deep search in potential local areas, and the second one avoids to search in poor areas and gives a higher possibility to searches to potential directions. Comparative experiments confirmed that the proposed strategies can improve the performance of EFWA significantly.

\section{Acknowledgment}

This work was supported in part by Grant-in-Aid for Scientific Research (15K00340) and the Natural Science Foundation of China (NSFC) under grant no. 61673025.

\section{REFERENCES}

[1] Tan, Y., and Zhu, Y.C., "Fireworks algorithm for optimization," Advances in Swarm Intelligence, vol. 6145 of the series Lecture Notes in Computer Science, pp. 355-364 (2010).

[2] Zheng, S.Q., Janecek A., and Tan, Y., "Enhanced Fireworks Algorithm," IEEE Int. Conf. on Evolutionary Computation, Cancun, Mexico, pp. 2069-2077 (2013).

[3] Liang, J., Qu, B., Suganthan P.N., and H., A., G., "Problem definitions and evaluation criteria for the CEC 2013 special session on realparameter optimization," Technical Report (2013). http://al-roomi. org/multimedia/CEC_Database/CEC2013/RealParameterOptimization/ CEC2013_RealParameterOptimization_TechnicalReport.pdf 